\title{
Intestinal Loop Evisceration after Vaginal Cuff Dehiscence: A Case Report and Review
}

Sonia De-Miguel-Manso ${ }^{1,2 *}$, Dakota Viruega-Cuaresma ${ }^{1}$, Elena García-García ${ }^{1}$, Carmen E. Badillo-bercebal ${ }^{1}$, Victoria Pascual-Escudero ${ }^{1}$, María López-País ${ }^{1}$.

${ }^{1}$ Servicio de Obstetricia y Ginecología, Hospital Clínico Universitario de Valladolid, Gerencia Regional de Salud de Castilla y León (SACYL), España.

${ }^{2}$ Departamento de Pediatría e Inmunología, Obstetricia y Ginecología, Nutrición y Bromatología, Psiquiatría e Historia de la Ciencia, Facultad de Medicina, Universidad de Valladolid, España.

*Corresponding author: Sonia De-Miguel-Manso, Servicio de Obstetricia y Ginecología, Hospital Clínico Universitario de Valladolid, Gerencia Regional de Salud de Castilla y León (SACYL), España

Received date: March 08, 2021; Accepted date: March 15, 2021; published date: March 24,2021

Citation: Miguel-Manso S D., Dakota V Cuaresma., Elena G García., Badillo-bercebal C E et all (2021) Intestinal Loop Evisceration After Vaginal Cuff Dehiscence: A Case Report And Review J. Women Health Care and Issues.4 (1); DOI: 10.31579/2642-9756/042.

Copyright: (c) 2021 Sonia De-Miguel-Manso, This is an open access article distributed under the Creative Commons Attribution License, which permits unrestricted use, distribution, and reproduction in any medium, provided the original work is properly cited.

\begin{abstract}
Background: Intestinal loop evisceration complicates 35-67\% of vaginal cuff dehiscence, constituting a medical emergency. In most cases, it is associated with genital prolapse in postmenopausal women with previous hysterectomy. Complications associated with the use of a pessary are rare. They are usually due to loss of patient follow-up or negligent use of the device.

Clinical presentation: We present the case of a 94-year-old patient who debuted with vaginal evisceration after vaginal cuff dehiscence, associated with prolonged use of pessary as a treatment for vaginal cuff prolapse and enterocele, after laparotomic hysterectomy. Vaginal repair was performed abdominally, due to the size and condition of the eviscerated loops, requiring intestinal resection. An omentum flap was attached to the vaginal cuff to improve healing and to try to occlude the Douglas space.

Conclusions: EV requires vaginal, abdominal, or mixed repair, generally deferring definitive prolapse treatment to a second stage and we recommend being very careful with the closure of the vaginal vault after any type of hysterectomy

Key Words: vaginal evisceration; vaginal cuff dehiscence; complications after hysterectomy; long-standing pessary
\end{abstract}

\section{Abbreviations}

VCD : vaginal cuff dehiscence

VE : vaginal evisceration

$\boldsymbol{\mu} \mathbf{L} \quad$ : microliter

Mg : milligram

DL : deciliter

PCR : protein C reactive

L : liter

G : gram

H : hour

\section{Introduction}

The vaginal cuff dehiscence (VCD) is the partial or complete separation of the anterior and posterior walls of the vaginal incision after a hysterectomy. It can be complicated by evisceration (VE), defined as the expulsion of intraperitoneal contents through this vaginal incision. Most commonly, this involves parts of distal ileum, but also omentum, appendix or fallopian tubes, and two cases of colon evisceration have been published [1].

The incidence of VCD ranges from $0.14 \%$ to $4.1 \%$, depending on the type of hysterectomy performed. Higher rates are reported after laparoscopic or robotic hysterectomy than after all other surgical techniques together [2].

VE complicates $35-67 \%$ of VCD, constituting a medical emergency. This event can generate complications, such as intestinal ischemia, sepsis and venous thrombosis [3]. $20 \%$ of patients will require bowel resection [4], $15 \%$ will have postoperative complications, and the mortality rate reaches $6 \%[3]$.

In most cases, VE due to VCD is associated with genital prolapse in postmenopausal women with previous hysterectomy [2]. 
On the other hand, complications associated with the use of a pessary to treat genital prolapse are rare. They are usually due to loss of patient follow-up or negligent use of the device. VCD, VE and vesico-vaginal or recto-vaginal fistulas are the main complications described [5].

We present the case of a 94-year-old patient with VE after VCD, associated with prolonged use of pessary as a treatment for vaginal cuff prolapse and enterocele, after total laparotomic hysterectomy.

\section{Clinical Presentation}

A 94-year-old patient came to the hospital emergency room for insidious onset abdominal pain in the previous days which suddenly became severe during the last 2 hours. She presented scant vaginal bleeding the day before.
The anamnesis was almost impossible due to her state of severe agitation because of the pain. As a medical history, she had hypertension, anxietydepressive syndrome and cognitive impairment. She also had two vaginal deliveries, menopause around the age of 50 and abdominal hysterectomy (indicated by prolapse) associated with cholecystectomy at the age of 60 . Subsequently, she suffered a recurrence (vaginal vault prolapse and enterocele) that was treated with a vaginal pessary for the last 8 years. Her last gynecological examination with pessary replacement was 6 months before the episode.

During the medical examination in the lithotomy position, it was striking the leakage of the intestinal contents outside the vulvar plane, with an intense red mesentery and signs suggestive of ischemia (Figure 1).

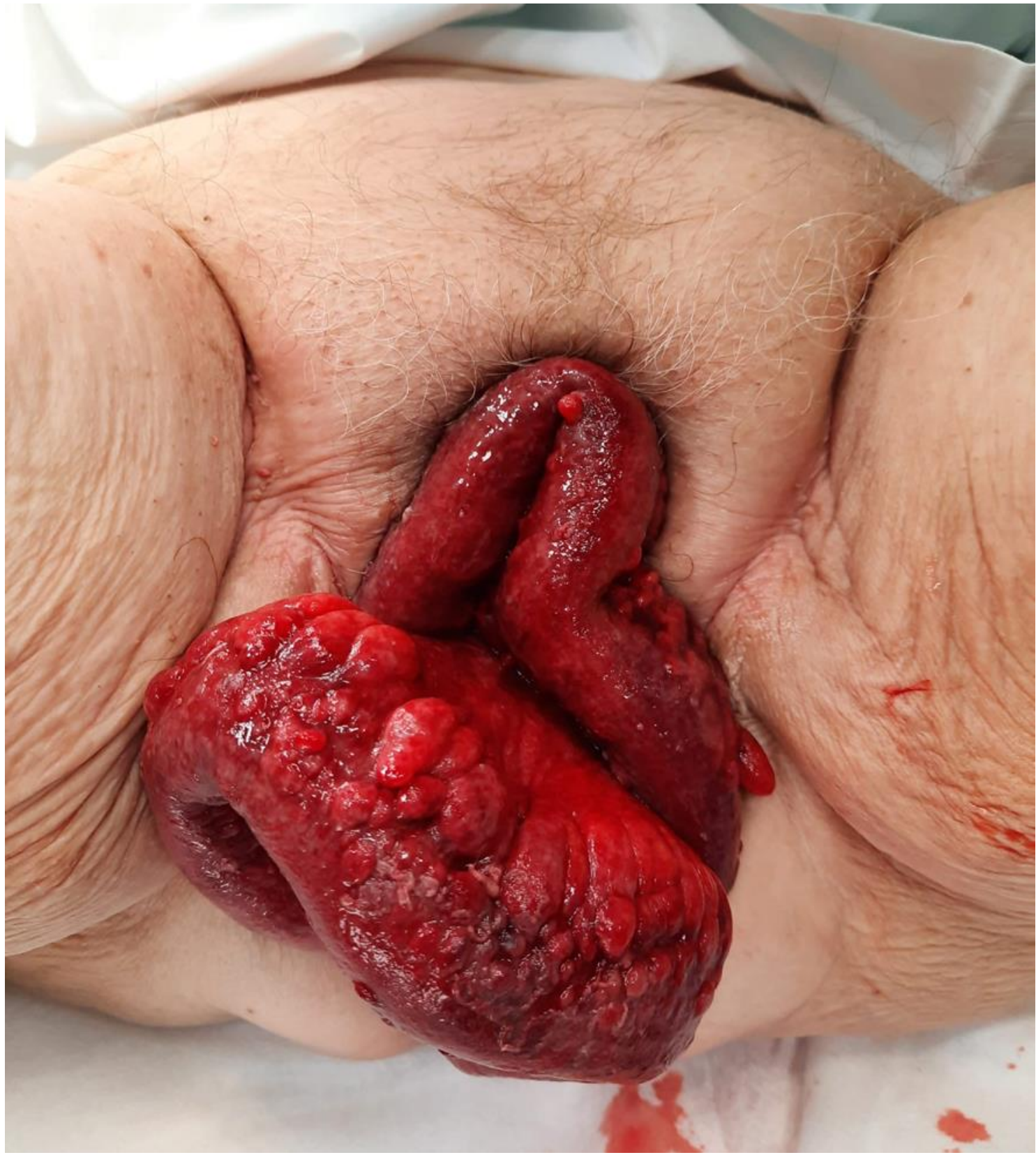

Figure 1: Vaginal evisceration: leakage of the intestinal contents outside the vulvar plane, with an intense red mesentery and signs suggestive of ischemia. 
On palpation, the abdomen was soft, but painful and with signs of peritoneal irritation. It wasn't possible vaginal examination to determine whether or not carrying the pessary.

Blood analysis revealed leukocytosis $\left(18.66 \times 10^{\wedge} / \mu \mathrm{L}\right)$ with a left deviation $(92.7 \%)$ and increased glucose $(214 \mathrm{mg} / \mathrm{dL})$ and protein $\mathrm{C}$ reactive (PCR $35 \mathrm{mg} / \mathrm{L}$ ). The rest of the parameters were normal: hemoglobin, hematocrit, sodium, potassium, creatinine and coagulation.

We contacted the General Surgery Service and a joint assessment of the patient was carried out; Urgent surgery was indicated due to the ischemic state of the intestinal loops, which was performed after receiving a negative PCR-Covid19 result. While the patient was transferred to the operating suite, the eviscerated intestinal contents were wrapped with moistened gauze in warm physiological saline solution; Intravenous broad-spectrum antibiotic therapy (amoxicillin-clavulanic acid $1 \mathrm{~g} / 8 \mathrm{~h}$ ) was started.
A subumbilical midline laparotomy was performed, releasing multiple intestinal adhesions.

After reaching the pelvis, the prolapsed intestinal content in the vagina was manually reduced. The vaginal pessary located in the posterior vaginal fornix was removed and the dehiscence of the vaginal vault was observed, with an extension of $3 \mathrm{~cm}$

The vagina was sutured by abdominal way, with late resorption thread $(0 / 0)$, with continuous and double suture, $1 \mathrm{~cm}$ from the vaginal edge. It didn't require debridement of the edges given its good appearance.

After this vaginal repair, the intestinal loops reintroduced to the abdomen were reviewed, showing a necrotic plaque and intense edema with signs of intestinal ischemia (Figure 2), which required the resection of $40 \mathrm{~cm}$ of the distal ileum.

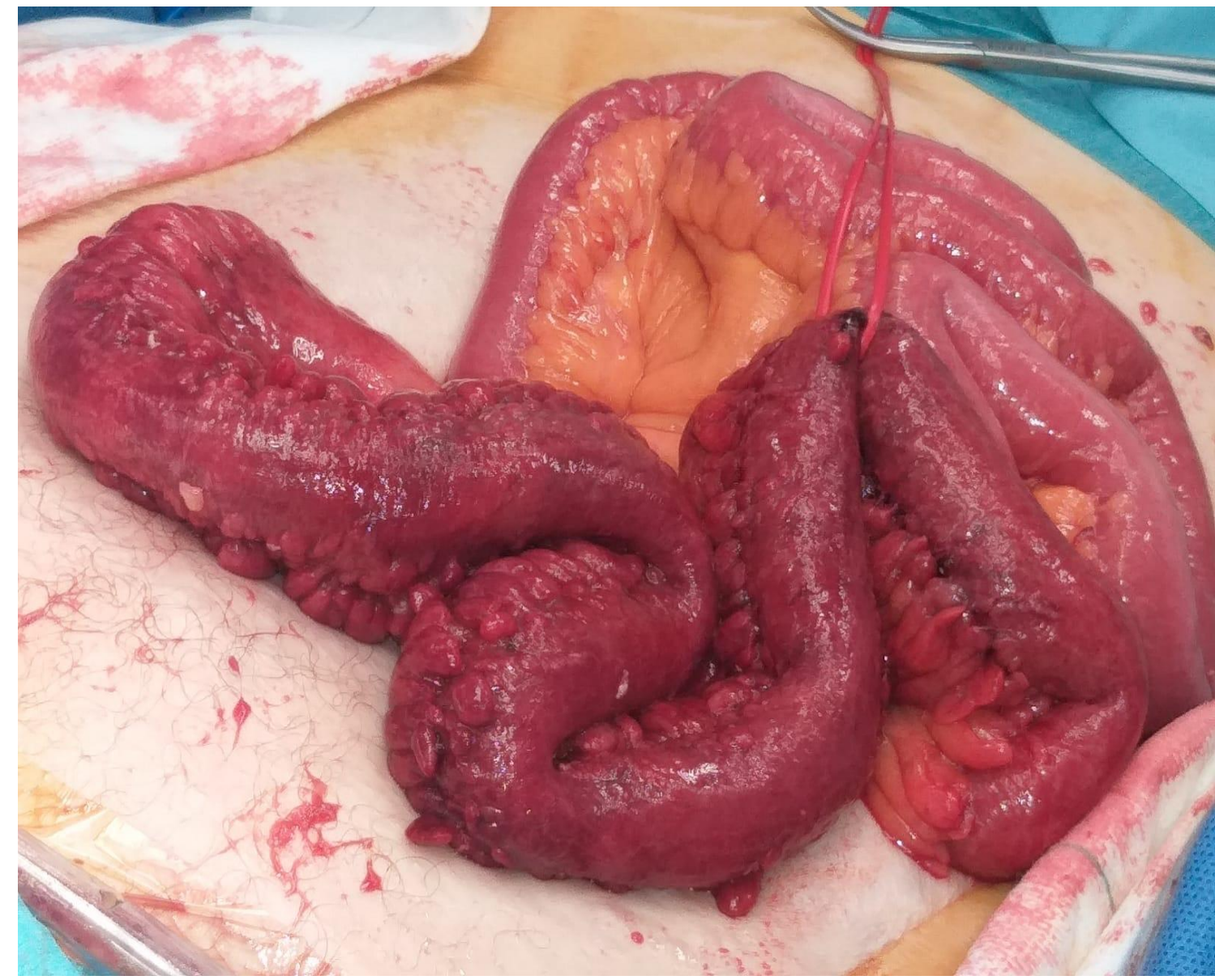

Figure 2: Intestinal loops reintroduced to the abdomen, showing a necrotic plaque and intense edema with signs of intestinal ischemia.

Finally, an omentum flap was approximated to the Douglas space, to suture it without tension to the vaginal cuff, with the intention of reinforcing the vaginal suture, given the characteristics of the patient (94 years and prolonged menopause).

The immediate postoperative period was favorable and on the seventh day the patient was discharged from the hospital.

The first gynecological examination was carried out at 3 months, verifying that there is no vaginal cuff prolapse or enterocele. New visits were scheduled; if the prolapse recurred, a new pessary would be avoided, recommending obliterative surgery in this patient.

\section{Discussion}

Historically, the majority of VCD cases were reported after vaginal hysterectomy, but distribution has changed with increasing laparoscopic hysterectomy. Possible factors are speculated: the surgeon's experience, errors in the technique, the lower safety of the stitches, the magnification of the image that would lead to sutures closer to the edges of the vaginal cuff and with less tissue, and the colpotomy performed with electrosurgery (thermal damage) [2].

Currently, the majority of VCD are associated with prolapse in postmenopause with a previous history of pelvic surgery. Especially when chronic medical conditions that transfer intra-abdominal pressure coexist, 
such as cough, asthma, gastroesophageal reflux and chronic constipation [2].

Other risk factors related to $\mathrm{VCD}$, in addition to menopause, due to vaginal atrophy, and hysterectomy are:

- Oncological pelvic surgery due to the weakness of tissues with tumor infiltration that are subjected to resection and adjuvant treatments such as radiotherapy and chemotherapy $[6,7]$.

- Colpotomy technique: the risk of VCD is greater if electrosurgery (thermal damage) is used. Ultrasonic energy is safer than mono and bipolar electrosurgery [2].

- Type of vaginal cuff closure. There seems to be a protective effect if the suture is performed with more than one layer versus a single layer [8] and with a bidirectional barbed suture [9]. On the other hand, there are no differences between 2 layers versus 1 cross layer [10] or between barbed and crossed non-barbed suture [11]

- Personal factors that affect the quality of the tissue and its healing, such as smoking, obesity, diabetes mellitus, genital prolapse, pelvic meshes, and chronic treatment with corticosteroids [2].

If there is an enterocele, the anatomy of the vaginal cuff is modified, which associated with atrophy, increases the risk of dehiscence with increased intra-abdominal pressure. In addition, in cases of severe vaginal cuff prolapse, venous stasis and pressure ulcers may appear [2].

In a premenopausal woman, the risk of vaginal cuff dehiscence with evisceration is low but is most frequently associated with vaginal trauma from early coitus after hysterectomy, a foreign body or gynecologic instrumentation [2].

Evisceration of the intestine through a VCD constitutes a surgical emergency. The most common symptoms are pelvic pain $(58-100 \%)$, vaginal bleeding (23.5-90\%), vaginal discharge (55.6\%), and signs of infection or peritoneal irritation [2].

The approach for surgical repair will depend on the clinical stability of the patient and the eviscerated bowel viability. In cases involving medical stable patients with a small amount of gutted intestine, without evidence of peritonitis or necrotic bowel, a vaginal approach could be performed [12], even associated with laparoscopy for inspection of intestinal loops. This mixed approach occurs in less than $15 \%$ of cases of VE [4]. Even the omentum can be identified in the vagina and reduced to the abdomen at the vaginal closure [4]

The rest of the clinical presentations must be repaired by transabdominal approach (laparoscopic or laparotomic) especially in cases of clinical or hemodynamic instability, large amount of eviscerated intra-abdominal content and suspected ischemia or incarceration/strangulation [3, 5].

The surgery will include vaginal closure, revision of intestinal loops reintroduced into the abdomen and possible resection in up to 20\% [4].

A definitive concomitant treatment of the prolapse is generally not applied, given the emergency of the process, but can be planned a posteriori [13]. Depending on the age and characteristics of the patient, the approach to the prolapse could be through repair surgery with sacrocolpopexy or fixation to the sacrospinous ligament, or obliterative surgery with colpocleisis.

Many types of vaginal repair have been reported with the aim of improving the closing conditions of the cuff and preventing recurrence of VCD with evisceration. They described below:

- Reconstruction of the rectovaginal septum by graciloplasty [14].
- Vaginal cuff fixation to the lateral abdominal wall [15].

- Omental flap attached to the vaginal cuff [4].

- Synthetic mesh. It is not a good option if the quality and vascularization of the tissue is poor $[2,4]$.

- Obliteration of the peritoneum of the Douglas space in case of enterocele [4] (Moschcowitz purse-string type suture [16].

In our case, VCD was associated with obesity, menopause, hysterectomy, vaginal cuff prolapse and enterocele, and use of pessary for 8 years (with adequate follow-up and last replacement 6 months before) as risk factors. During the surgical procedure, the pessary was found attached to the posterior vaginal wall. This may suspect that the enterocele altered vaginal angulation, which, associated with atrophy and increased intraabdominal pressure, caused the rupture of the vaginal suture. The surgical repair way was abdominal due to the patient's clinical condition (severe pain with laboratory involvement) and the state of the eviscerated bowel, which would probably require surgical resection.

Presurgical management was carried out according to updated bibliographic recommendations [12,13], in relation to wrapping the intestinal loops with compresses soaked in warm physiological saline and initiating broad-spectrum prophylactic antibiotic therapy.

Before cuff repair, the need to debride the cuff edges was assessed, as indicated by some publications $[2,3]$, but it was not necessary due to the absence of signs of necrosis or infection.

The cuff closure was performed with continuous double layer suture $(0 / 0)$, $1 \mathrm{~cm}$ from the edge of the cuff, technique that appears to be protective with respect to the recurrence of VCD [2].

Given the age of the patient, 94 years and her clinical emergency status, definitive treatment for the prolapse was not performed; the use of synthetic meshes was not considered either. An omentum flap was released to fix it to the vaginal vault, with the double intention of occluding the Douglas space (and avoid enterocele) and improve vascularization during healing.

Rubin et al., in an 82-year-old patient with VCD and VE after vaginal histerectomy at the age of 72 and continued use of a pessary, performed the inspection of the intestinal loops by laparotomy and concomitant treatment of prolapse with colpocleisis [5].

Other authors have published cases of VE after pessary use, associated with menopause, vaginal and abdominal hysterectomy [5]. In 1976, Thiele described a case of VCD and VE after negligent use of the pessary for 4 years without revisions [17].

When genital prolapse is corrected with a pessary, in addition to selecting the size properly, patients and their caregivers should be instructed in its correct management. Pessary designs and materials could be improved and consultation time should be devoted to teaching insertion and removal techniques at home.

On the other hand, a review regarding the use of vaginal estrogens to prevent VCD, both before and after hysterectomy, are not conclusive [13].

\section{Some premises are recommended to prevent vcd:}

- Preoperative treatment of modifiable risk factors such as vaginal infections [18] or vaginal estrogen therapy 6 weeks before [19].

- Prophylactic antibiotic therapy prior to skin incision, although hysterectomy is considered clean surgery [20].

- Preoperative asepsis. On the skin, the use of alcoholic chlorhexidine has shown significant superiority over povidone iodine in preventing superficial and deep infections at the 
incision site. In the vagina, the aqueous chlorhexidine solution is highly effective in reducing the bacterial count [20].

- Type of hysterectomy. Higher risk of VCD in laparoscopic versus vaginal approach [21] and also in radical versus simple hysterectomy [22].

- Colpotomy technique. If electrosurgery is used, it is recommended to minimize lateral extension and suture distally of thermal damage [2].

- Amount of sutured tissue. Especially important in the closure of the vaginal cuff by laparoscopy. Thus, O'Hanlan [23] recommends a $5 \mathrm{~mm}$ interval between sutures with a depth of 5 $\mathrm{mm}$ and Kho [24] recommends a separation of at least $5 \mathrm{~mm}$ from the coagulated tissue. Nezhat et al. [25] increase the amount of tissue, incorporating at least 1.5, which implies a greater dissection of the vesicovaginal space.

- Continuous or interrupted vaginal closure suture. Continuous suturing has been shown in several studies to reduce the risk of VCD and VE [10, 11].

- One or two-layer closure. Several authors have demonstrated the superiority of the two-layer closure in laparoscopic hysterectomy [26, 27].

- $\quad$ Suture material. Few publications include direct comparisons between types of sutures. There are several studies on absorbable barbed sutures introduced in 2009, which are characterized by a longer duration of tension force compared to braided sutures (90-180 days versus 28 days). Thus, several authors have shown to reduce the risk of VE with bidirectional barbed suture [11, 27].

- Avoid complications such as infections, bleeding or bruising of the vaginal vault, as they can increase the risk of dehiscence [2].

- $\quad$ Postoperative management. It seems appropriate to wait at least 6 weeks to restart sexual intercourse. If there are several risk factors for VCD, it may be reasonable to extend the period to 12 weeks [28]. It is very important to avoid postoperative constipation to reduce the tension of the vaginal vault during Valsalva in the healing process.

\section{Conclusions}

- Most VCD are associated with women in menopause, a history of hysterectomy, and a chronic increase of intra-abdominal pressure. If we add to this the prolonged or negligent use of a pessary, the probability of VCD increases.

- Although VCD is an infrequent complication, when it occurs, it is associated with VE in up to $35-67 \%$ of cases.

- VE constitutes a medical emergency, which requires vaginal, abdominal or mixed repair, generally deferring the definitive treatment of the prolapse to a second stage.

- We recommend being very careful with vaginal vault closure after any type of hysterectomy, avoiding electrosurgery, infection and bleeding, performing the colporrhaphy with bidirectional barbed suture or two layers with braided suture and facing enough tissue at the suture.

\section{Acknowledgements}

The authors thank the Department of General Surgery for collaboration.

\section{Conflict of Interest: None.}

\section{Funding Information}

No funding was obtained for the implementation of this study.

\section{References:}

1. Serati M, Casarin J, Braga A. Transvaginal repair of a misunderstood large bowel evisceration six months after vaginal hysterectomy. Eur J Obstet Gynecol Reprod Biol. 2017 Oct; 217:180-181.

2. Nezhat C, Burns MK, Wood M, Nezhat C, Nezhat A, Nezhat F. Vaginal Cuff Dehiscence and Evisceration: A Review. Obstet Gynecol. 2018 Oct;132(4):972-985.

3. Rogers P, Lee H, Jape K, Zi Qin Ng, Koong D. Vaginal evisceration of small bowel. J Surg Case Rep. 2019 Nov 20;2019(11):rjz317.

4. Narducci F, Sonoda Y, Lambaudie E, Leblanc E, Querleu D. Vaginal evisceration after hysterectomy: the repair by a laparoscopic and vaginal approach with a omental flap. Gynecol Oncol. 2003 Jun;89(3):549-551.

5. Rubin R, Jones KA, Harmanli OH. Vaginal evisceration during pessary fitting and treatment with immediate colpocleisis. Obstet Gynecol. 2010 Aug;116 Suppl 2:496-498.

6. Ceccaroni M, Berretta R, Malzoni M, Scioscia M, Roviglione G, Spagnolo E, et al. Vaginal cuff dehiscence after hysterectomy: a multicenter retrospective study. Eur J Obstet Gynecol Reprod Biol. 2011 Oct;158(2):308-313.

7. Drudi L, Press JZ, Lau S, Gotlieb R, How J, Eniu I, et al. Vaginal vault dehiscence after robotic hysterectomy for gynecologic cancers: search for risk factors and literature review. Int J Gynecol Cancer. 2013 Jun;23(5):943-950.

8. Fanning J, Kesterson J, Davies M, Green J, Penezic L, Vargas $\mathrm{R}$, et al. Effects of electrosurgery and vaginal closure technique on postoperative vaginal cuff dehiscence. JSLS. Jul-Sep 2013;17(3):414-417.

9. Siedhoff MT, Yunker AC, Steege JF. Decreased incidence of vaginal cuff dehiscence after laparoscopic closure with bidirectional barbed suture. J Minim Invasive Gynecol. MarApr 2011;18(2):218-223.

10. Jeung C, Baek JM, Park EK, Lee HN, Kim CJ, Park TC, et al. A prospective comparison of vaginal stump suturing techniques during total laparoscopic hysterectomy. Arch Gynecol Obstet. 2010 Dec;282(6):631-638.

11. Bogliolo S, Musacchiv V, Dominoni M, Cassani C, Gaggero $\mathrm{CR}$, De Silvestri A, et al. Barbed suture in minimally invasive hysterectomy: a systematic review and meta-analysis. Arch Gynecol Obstet. 2015 Sep;292(3):489-497.

12. Matthews CA, Kenton $\mathrm{K}$. Treatment of vaginal cuff evisceration. Obstet Gynecol. 2014 Oct;124(4):705-708.

13. Hur HC, Lightfoot M, McMillin MG, Kho KA. Vaginal cuff dehiscence and evisceration: a review of the literature. Curr Opin Obstet Gynecol 2016;28:297-303.

14. Halwani Y, Nicolau-Toulouse V, Oakes J, Leipsic J, Geoffrion $\mathrm{R}$, Wiseman SM. Transvaginal strangulated small intestinal hernia after abdominal sacrocolpopexy: case report and literature review. Hernia. 2013 Apr;17(2):279-283.

15. Cruickshank L, Amin A, DeSilva A. Small bowel evisceration: a late complication following vaginal hysterectomy. J Obstet Gynaecol. 2018 Jul;38(5):733.

16. Hirsch, Käser, Iklé (2000). Cirugía vaginal en prolapsos pélvicos. In Atlas de cirugía ginecológica (3. a ed., p. 279). Madrid: Marban. Madrid: Marban.

17. Thiele H, Pampel R. Vaginal rupture with prolapse of the small intestine. Zentralbl Gynakol. 1976;98(15):948-949.

18. Soper DE, Bump RC, Hurt WG. Bacterial vaginosis and tricomoniasis vaginitis and risk factors for cuff cellulitis after adbominal hysterectomy. Am J Obstet Gynecol 1990;163:1016-1021. 
19. Rahn DD, Good MM, Roshanravan Sm, Shi H, Schauffer JI, Singh RJ, et al. Effects of preoperative local estrogen in postmenopausal women with prolpapse: a randomized trial. J Clin Endocrinol Metab 2014;99:3728-3736.

20. Prevention of Infection After Gynecologic Procedures. ACOG Practice Bulletin No. 195. American College of Obstetricians and Gynecologists. Obstet Gynecol. 2018 Jun;131(6):e172e189.

21. Hur HC, Donnellan N, Mansuria S, Barber RE, Guido R, Lee T. Vaginal cuff dehiscence after different modes of hysterectomy. Obstet Gynecol. 2011 Oct;118(4):794-801.

22. Nick AM, Lange J, Frumovitz M, Soliman PT, Schmeler KM, Schlumbrecht MP, et al. Rate of vaginal cuff separation following laparoscopic or robotic hysterectomy. Gynecol Oncol. 2011 Jan;120(1):47-51.

23. O'Hanlan KA, Emeney PL, Peters A, Sten MS, McCutcheon SP, Struck DM, Hoang JK. Analysis of a Standardized Technique for Laparoscopic Cuff Closure following 1924 Total Laparoscopic Hysterectomies. Minim Invasive Surg. 2016;2016:1372685.
24. Kho RM, Akl MN, Cornella JL, Magtibay PM, Wechter ME, Magrina JF. Incidence and characteristics of patients with vaginal cuff dehiscence after robotic procedures. Obstet Gynecol. 2009 Aug;114(2 Pt 1):231-235.

25. Nezhat C, Grace LA, Razavi GM, Mihailide C, Bamford H. Reverse Vesicouterine Fold Dissection for Laparoscopic Hysterectomy After Prior Cesarean Deliveries. Obstet Gynecol. 2016 Sep;128(3):629-633.

26. Cronin B, Sung VW, Matteson KA. Vaginal cuff dehiscence: risk factors and management. Am J Obstet Gynecol. 2012 Apr;206(4):284-288.

27. Nawfal AK, Eisenstein D, Theoharis E, Dahlman M, Wegienka G. Vaginal cuff closure during robotic-assisted total laparoscopic hysterectomy: comparing vicryl to barbed sutures. JSLS. Oct-Dec 2012;16(4):525-529.

28. Hur HC, Guido RS, Mansuria SM, Hacker MR, Sanfilippo JS, Lee TT. Incidence and patient characteristics of vaginal cuff dehiscence after different modes of hysterectomies. J Minim Invasive Gynecol. 2007 May-Jun;14(3):311-317.

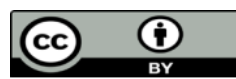

This work is licensed under Creative Commons Attribution 4.0 License

To Submit Your Article Click Here: Submit Manuscript

DOI: $10.31579 / 2642-9756 / 042$
Ready to submit your research? Choose Auctores and benefit from:

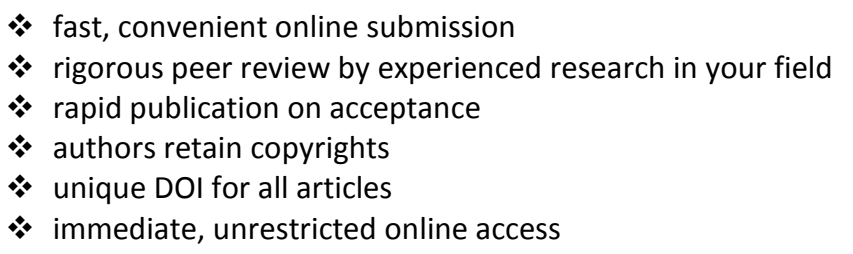

At Auctores, research is always in progress.

Learn more www.auctoresonline.org/journals/women-health-care-andissues 